EOMmunutriln? Communication et organisation

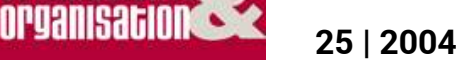

Les vallées : sens, territoires et signes

\title{
Des vallées en Eure et Loir
}

\section{Florence Hénon}

\section{OpenEdition}

Journals

Édition électronique

URL : http://journals.openedition.org/communicationorganisation/2952

DOI : 10.4000/communicationorganisation.2952

ISSN : 1775-3546

\section{Éditeur}

Presses universitaires de Bordeaux

\section{Édition imprimée}

Date de publication : 2 octobre 2004

ISSN : 1168-5549

\section{Référence électronique}

Florence Hénon, « Des vallées en Eure et Loir », Communication et organisation [En ligne], 25 | 2004, mis en ligne le 27 mars 2012, consulté le 02 mai 2019. URL : http://journals.openedition.org/ communicationorganisation/2952 ; DOI : 10.4000/communicationorganisation.2952

Ce document a été généré automatiquement le 2 mai 2019

(c) Presses universitaires de Bordeaux 


\title{
Des vallées en Eure et Loir
}

\author{
Florence Hénon
}

1 Depuis quelques années, les ternies de pôles, vallées, réseaux, filières, se lisent dans des revues spécialisées et descendent dans la rue. Lorsque nous utiliserons ces termes, il s'agira, uniquement, d'un groupement thématique d'entreprises c'est-à-dire, pour nos quatre exemples, quatre associations ayant chacune un thème bien défini : parfum et cosmétique/Chimie fine/Bois/Agriculture et agroalimentaire. C'est donc autour de chacun de ces sujets que se regroupent différents acteurs économiques fait partie d'une étude et d'une analyse en cours d'élaboration que je réalise actuellement à propos des mises réseaux en Eure-et-Loir. En quête du sens, je propose, avec cet article, un travail sur l'identité qui s'articulera autour des différents éléments visuels et non visuels qu'émettent ces quatre pôles à travers leurs communications. Racines historiques et géographiques, sources d'identité, formes de communication et signes visuels, que sont les logos et le site internet de la Cosmetic Valley seront exposés dans le travail qui suit.

2 Le CODEL, Comité de Développement Economique d'Eure-et-Loir a pour missions de créer des emplois dans le département, d'implanter des entreprises, de favoriser le développement des entreprises, de conforter la création d'entreprise et d'animer des réseaux d'entreprises. Le Codel et le conseil général apportent leur appui au développement économique local et au regroupement de compétences dans le cadre de filières comme la Cosmetic Valley, Polepharma, Perchebois et/ou Agrodynamic. L'identité collective de ces groupements révèle la volonté de tirer partie de nouvelles opportunités de communication. Le Codel, dirigé par Jean-Luc Ansel, directeur lui-même de la Cosmetic Valley, de Polepharma et de Perchebois est à l'origine de la création de ces quatre réseaux thématiques. S'il est bien évident que seule, une équipe solide et volontaire, avec différents acteurs économiques peut réussir à construire un pôle de compétitivité, la personnalité de Jean-Luc Ansel joue un rôle essentiel et incontestable dans ces quatre réalisations. Ingénieur bois, il a dirigé différents groupes industriels autour du bois, enseigné à l'école supérieure du bois à Paris, consultant international, spécialisé dans le bois et depuis 1989, il pilote de Codel. Homme d'expériences dans les domaines publics et privés, il est apprécié pour ses compétences, son sens du contact, son dynamisme et ses 
capacités à manager. Depuis 1989, Jean-Luc Ansel a donné à la fois au Codel et au Conseil Général d'Eure-et-Loir, une très forte réputation en matière d'intervention économique et d'implantation.

\section{Identité}

3 L'identité est un concept d'émission qui spécifie le sens et le projet d'une marque. « La connaissance de l'identité, paradoxalement, redonne une certaine liberté d'expression car elle rappelle la prééminence du fond sur les stricts aspects formels. L'identité de marque définit ce qui doit rester permanent et ce qui peut évoluer, varier ».

\section{Racines historiques et géographiques des pôles industriels}

4 Les quatre pôles qui font l'objet de notre étude sont attachés à un lieu géographique précis du département d'Eure et Loir voire pour Agrodynamic, le département dans son ensemble. Chartres pour la Cosmetic Valley, Dreux pour Polepharma, Nogent-le-Rotrou pour Perchebois.

5 L'histoire d'une vallée révèle et participe aux fondements de son identité. De cette façon, elle devient le capital des valeurs qui construisent le projet et la vision de la vallée. Pour mieux comprendre l'émergence de tel ou tel pôle de compétitivité, retrouver l'origine de celui-ci permet de mieux appréhender une analyse de la vallée. Ainsi, les pôles que nous trouvons en Eure et Loir ne se sont pas créées par hasard.

6 L'histoire de la Cosmetic Valley débute dans les années 60/70 lorsque le parfumeur Fabergé implante ses équipes de recherche et de production à Chartres, sans doute pour des raisons financières. Elle se poursuit avec l'arrivée de Lancaster qui y installe son site européen en 1972. En 1974, Jean-Paul Guerlain décide de décentraliser son lieu de production de Courbevoie à Chartres. En 1976, c'est le tour de Paco Rabanne...

7 À partir de ce moment-là, de nombreuses PME s'installent autour des grandes marques et opèrent en amont (laboratoires de matières premières, plasturgistes, concepteurs, fabricants de cosmétiques..). Mais l'histoire de ce réseau industriel est aussi et surtout celle d'une rencontre entre deux hommes, passionnés et de fortes personnalités : JeanPaul Guerlain et Jean-Luc Ansel. Attaché à la vallée de l'Eure, située à 5 kilomètres de Chartres, Jean-Paul Guerlain possédait une résidence secondaire. Travail, amitié et loisir se sont fortement conjugués et ont donné naissance à la Cosmetic Valley, que le premier préside et le second dirige.

8 La première appellation, en 1994, fut « Association Parfums Cosmétiques » puis, en 1998, elle se transforma, pour des raisons d'impact international, en "Cosmetic Valley » qui nous rappelle, bien évidemment, la Silicon Valley et « Chartres, la capitale « en sous titre. Par ces termes, la ville signe sa nouvelle politique de communication : Chartres, ville du parfum et de la lumière (fête de la lumière, cathédrale). Cette appellation mise sur la stratégie de développement économique, et mondial d'industriels de la filière parfums et cosmétiques axe prioritaire mais aussi internationale. Premier réseau français, la Cosmetic Valley regroupe, à Chartres et dans le département d'Eure-et-Loir, une concentration impressionnante d'entreprises, la plus forte densité d'établissements de ce 
secteur. Près de 100 entreprises et 6000 professionnels assurent une grande partie de la production de l'industrie du luxe et de la beauté. Cela représente $70 \%$ des entreprises de la filière.

Polepharma représente l'industrie pharmaceutique en Eure et Loir avec Dreux comme capitale. Premier producteur européen de médicaments devant l'Allemagne et la GrandeBretagne, Dreux se situe à l'intersection de trois régions: Centre, Ile-de-France et Normandie et Polepharma figure l'un des tout premiers réseaux européens de la filière pharmaceutique. De grands noms : Abbott, Beaufour-Ipsen, Glaxo, Lacteol, Léo, Norgine Pharma etc...

Association professionnelle, créée en juin 2002, la filière Polepharma est dirigée par JeanLuc Ansel et présidée par M. Marc Ivacheff, directeur des laboratoires Norgine Pharma. «L'ambition de Polepharma est d'encourager le maillage des compétences et les synergies, de développer des courants d'affaires au sein d'un réseau, de mettre en place des services adaptés à la filière, d'améliorer la formation, de promouvoir le savoir-faire des entreprises sur les salons professionnels et de favoriser l'implantation de nouvelles entreprises «. Une centaine d'entreprises et quelques 15000 professionnels participent au bon fonctionnement de ce réseau. D'un bout à l'autre de la chaîne, une main d'œuvre de qualité et tous les savoir-faire s'expriment, développement de formulations, tests analytiques, fabrication de formes et de dosages, conditionnement, marketing, fabrication de médicaments, emballage, PLV, logistique...

Perchebois est née à Nogent le Rotrou d'une étude lancée à la demande du Codel et de la chambre des métiers. Cette dernière a mis en évidence à la fois les ressources magnifiques des forêts d'Eure et Loir et du Perche et un savoir-faire ancestral qu'il ne fallait surtout pas perdre. Créée fin 1997 et présidée par Bernard Dordoigne, cette association, loi 1901 veut valoriser à la fois la tradition et ses produits, sciage, planches, merrains, charpentes, menuiseries percheronnes et meubles de région.

Première région d'Europe et premier département céréalier, la Beauce, le grenier de la France et de surcroît l'Eure et Loir est géographiquement destiné à créer le pôle industriel Agrodynamic. Née de la volonté commune du Codel, de la chambre d'Agriculture, des industriels et des collectivités, Agrodynamic, en cours de lancement veut valoriser les savoir-faire et les potentialités des professionnels de l'agriculture et de l'agro-industrie.

\section{Les sources d'identité}

Les vallées ont un projet, une volonté, une visée qui se trouve malheureusement, très rarement écrit. Cependant, Polepharma comme Perchebois et les autres vallées d'Eure et Loir laissent leurs empreintes et donc des signes qui permettent de décrypter la spécificité de chacune d'entre elles, leur contenu et leurs valeurs. Nous notons à la fois des éléments communs à toutes les quatre et quelques spécificités. L'unicité et la spécificité représentent les meilleurs indices pour définir et surtout comprendre le pôle. Le produit ou le service est la première source d'identité du pôle car ce dernier y injecte ses valeurs. Produits et services sont donc porteurs de sens.

L'attachement et les racines géographiques de ces quatre pôles participent à leurs sources d'identité, à leurs particularités. De cette façon, ces pôles s'approprient des valeurs liées à la source départementale voire régionale. Ainsi, la situation géographique de la Cosmetic 
Valley, à $60 \mathrm{~km}$ de Paris représente un atout évident et stratégique. La proximité de la capitale n'est pas le seul intérêt car d'importants axes routiers existent et facilitent de ce fait les liaisons (autoroute A11 et nationale 10). Ce que nous appelons communément la 3e couronne parisienne symbolise une véritable opportunité au Sud-Ouest de Paris. En effet, Chartres et son département offrent des terrains industriels, très diversifiés à des prix compétitifs trois fois moins élevés qu'en région parisienne (terrain en pleine campagne, espace vitrine au bord de l'autoroute Océane qui draine plus de 13 millions de véhicules tous les ans). Cette concentration de clients et d'acteurs d'une filière, sur un même espace géographique valorise non seulement la dynamique de la Cosmetic Valley mais aussi les synergies entre les membres du réseau. «Bénéficier de ressources humaines qualifiées et offrir un cadre de vie de qualité «sont autant d'atouts qu'offre cette association professionnelle.

Perchebois se sert des chênes et des fruitiers nombreux du Perche et de la spécificité rurale comme éléments de base à toute émission de communication.

Agrodynamic allie tradition agricole de la Beauce, innovation industrielle et recherche.

Les valeurs communes que les quatre pôles expriment nettement sont :

achement au passé, aux racines, aux savoir-faire locaux, au territoire et à l'avenir du département. Un réseau de compétences et de savoir-faire du métier se concentre dans ces associations.

- La solidarité. Jean-Luc Ansel, directeur du Codel nous explique que la volonté des dirigeants est de " fédérer des gens entre eux » et de mettre en place " un réseau de soustraitants autour des grands noms ", pour la Cosmetic Valley, par exemple. Adhérent de l'association Perchebois, Hubert Germond explique: «j'ai adhéré à Perchebois pour rompre l'isolement, car, parfois, nous les artisans, nous sommes bien seuls et isolés de la demande. C'est pour cela que le travail sur l'image de l'association et de ses adhérents me semble important. ».

- L'éthique et la confiance Instaurer un code moral, un respect et favoriser une «solidarité entre les sous-traitants «tel est le discours du directeur du Codel. La réalisation d'une charte de qualité, pilotée par la chambre des métiers a permis d'encadrer et de valoriser le savoir-faire dont Perchebois et ses adhérents font la promotion. Cette charte de qualité définit les règles de fabrication des mobiliers traditionnels et contemporains, avec estampillage à la clé.

21 - La convivialité. Initier des rencontres professionnelles et des conventions d'affaires de niveau international, organiser des rendez-vous B to B par l'intermédiaire de la convention d'affaires "Cosmetech Meetings", chaque septembre à Chartres, tel est un des engagements de la Cosmetic Valley. La formule " Cosmetech « semble efficace car elle privilégie contacts personnalisés et confidentialité. Tous les secteurs de la filière y sont représentés et les grands prestataires français et européens sont au rendez-vous tous les ans pendant deux jours. En simultanéité, sur le même lieu et de façon annuelle se déroule la convention "Chiminov » (chimie fine) et à Dreux, à 30 minutes de Chartres, celle de «Polepharma ». Cela permet aux visiteurs, de cumuler des rencontres professionnelles complémentaires car certaines entreprises travaillent à la fois avec la Cosmetic Valley \& Polepharma.

22 Représentation exhaustive de toute une filière, priorité donnée aux contacts $B$ to $B$, territoire d'accueil privilégié, réseau industriel doté d'une forte dynamique, ressources humaines qualifiées, situation géographique et un cadre de vie de qualité, telles soin 
l'image et la stratégie de communication de la Cosmetic Valley. En 200 à compléter, la DATAR, Délégation à l'Aménagement du Territoire et,i l'Action Régionale, lui a attribué le label SPL, Système Productif Localisé. C'est à ce jour le seul des quatre pôles a l'avoir obtenu. Perchebois et Polepharma espèrent l'obtenir prochainement. La convivialité se gère en interne et en externe du groupe lui-même.

Pour associer tous les euréliens à la dynamique de la filière économique phare du département, la Cosmetic Valley organise depuis septembre 2001 et cela tous les ans en septembre, une fête du parfum qui dure une semaine. Elle renforce l'action des conventions d'affaires, citées plus haut, qui, elles, s'adressent davantage aux professionnels. Pour éveiller les sens, cet événement regroupe des visites, des expositions, une vente aux enchères, un salon des collections parfumées..

Après la terrible tempête de 1999, le secteur bois est devenu prioritaire et un forum international du bois, le premier en son genre, a montré la volonté de la politique du commerce extérieur de la région. Professionnels français, acheteurs Scandinaves, britanniques, autrichiens et marocains, l'offre et la demande se sont rencontrées.

La première semaine du bois s'est déroulée du 24 au 31 mars 2001 à Nogent-le-Rotrou réunissant les professionnels du bois avec une porte ouverte au publique et de nombreuses démonstrations. Pierre-Marie Desclos a donné, à cette occasion, une conférence sur l'avenir du secteur bois face à la mondialisation. Depuis, tous les ans, à la même période, la semaine du bois propose différentes manifestations, conférences, portes ouvertes spéciales scolaires, exposition...

Les adhérents ont signé un partenariat avec le Parc du Perche et ils ont développé avec le designer Sylvie Hébert une gamme de mobilier typique et contemporain du Perche.

L'association a une convention avec la Société des Experts Bois (SEB) pour pérenniser et concrétiser les relations déjà établies et pour associer les deux structures dans les salons et expositions en France et à l'étranger. Participation aux salons français (Nantes, Paris et Nogent-le-Rotrou) et étrangers: Interzum à Cologne en Allemagne, à Valence et à Shanghaï. Perchebois travaille aussi à une plate-forme de conditionnement des déchets bois en vue de les exploiter pour le chauffage.

Jean-Luc Ansel affirme que ces quatre pôles sont aussi du plaisir de faire des choses ensembles (rendez-vous mensuels, salons..) et de construire des pôles de compétitivité pour l'avenir du département et des euréliens.

\section{Deux formes de communication}

Le Codel gère la communication de chacune de ces associations et elles ont toutes un relais personnifié au sein même du Codel.

Les pôles, en matière de communication utilisent principalement deux niveaux de discours : la communication d'entreprise et la communication d'institution.

La première a une vocation de transparence et elle révèle le physique de l'association, ses moyens humains, technologiques et financiers. Elle met en valeur les ambitions, les objectifs et les moyens que le pôle met à disposition. Le contenu de cette communication doit être factuel et économique. L'exactitude et la véracité sont les critères d'obligation. Valoriser les savoir-faire et faire connaître l'Eure et Loir, la région sont certains de leurs objectifs. La seconde expose les valeurs du pôle ce qui lui sert à légitimer son activité. 


\section{La communication d'entreprise de la Cosmetic Valley.}

l'économie percheronne. Futaies de chêne, haies et bocages de bois fruitiers offrent aux industriels et aux artisans une matière première de haute qualité. L'ensemble de la chaîne, de la plantation des arbres au produit fini, l'exploitation de la forêt se divise en deux périodes. Une première transformation regroupe l'abattage, le débusquage et le débardage pour les activités les plus visibles et sont le résultat d'un long travail des sylviculteurs. La seconde élaboration concerne les meubles traditionnels et contemporains et la menuiserie. Cette deuxième partie du travail du bois représente 200 emplois dans l'artisanat et 360 dans l'industrie. Les métiers de la première et deuxième transformation du bois sont les exploitants forestiers, les scieurs, les menuisiers, les ébénistes, les architectes et un centre de formation. Promotion des essences secondaires comme l'acacia. En 2002, le syndicat des propriétaires forestiers sylviculteurs d'Eure et Loir a rejoint Perchebois. Bertrand de Rougé

De la culture de l'angélique et plantes aromatiques fournissant des extraits naturels pour les cosmétiques, au conditionnement et à l'expédition des produits finis, en passant par la composition de nouveaux jus, l'injection plastique et l'habillage des flacons, la création de formules cosmétiques, le contrôle et l'évaluation des produits, la conception de PLV, la logistique, tous les secteurs de la filière sont présents. De grands noms de parfums classiques, Shalimar, Habit Rouge, XS mais aussi les succès des dernières saisons, Lolita Lempicka, Boudoir, Ultraviolet etc..

d'agglomération de Chartres assurent le développement de la Cosmetic Valley. Ils apportent à la fois une assistance économique et le soutien d'experts pour la conduite des projets d'investissements (études d'implantation, solutions de financement..). Grâce à ses partenaires publics, la Cosmetic Valley propose des structures d'accueil facilitant les nouvelles implantations.

Présente sur les grands salons internationaux (Bologne, Hong Kong, Dubaï, Moscou, Tokyo..), la Cosmetic Valley se veut le porte-drapeau de la « french touch». Elle s'engage à : « aller à la rencontre des professionnels du monde entier, participer au rayonnement du « Made in France » établir des relations d'affaires et développer les activités exporl des entreprises de la filière ».

de transformation dans la filière bois du Perche c'est-à-dire : « création d'une identité du meuble et de la menuiserie du Perche, promotion du savoir faire local des artisans et industries du bois, création d'un groupe cohérent de références pour la filière bois ». La réalisation d'une charte de qualité, pilotée par la chambre des métiers a permis d'encadrer et de valoriser le savoir-faire dont Perchebois et ses adhérents font la promotion. Cette charte de qualité définit les règles de fabrication des mobiliers traditionnels et contemporains, avec estampillage à la clé.

L'association a une convention avec la Société des Experts Bois (SEB) pour pérenniser et concrétiser les relations déjà établies et pour associer les deux structures dans les salons et expositions en France et à l'étranger. Participation aux salons français (Nantes, Paris et 
Nogent-le-Rotrou) et étrangers: Interzum à Cologne en Allemagne, à Valence et Shanghaï.

Perchebois travaille à une plate-forme de conditionnement des déchets bois en vue de les exploiter pour le chauffage.

\section{Les prismes d'identité des quatre filières euréliennes.}

Le prisme d'identité avec ses six facettes, le physique, la personnalité, la relation, la culture, le reflet et la mentalisation, participe à l'élaboration, à la définition de l'identité de chaque pôle. De cette façon, le prisme forme un tout structuré qui marque son territoire de potentialités.

41 Le physique est la base de la marque, la colonne vertébrale qui forme la valeur ajoutée du pôle. Il rassemble l'ensemble des caractéristiques objectives plus ou moins latentes. La personnalité correspond au caractère de la vallée.

43 À chaque groupement thématique d'entreprises correspond une culture, un univers culturel, c'est-à-dire un système de valeurs, qui est essentiel. La relation, comme l'explique Kapferer, est une transaction entre personnes, un échange.

Le reflet l'image de l'utilisateur auquel s'adresse la vallée. La cible la description des utilisateurs du pôle. La mentalisation le miroir interne

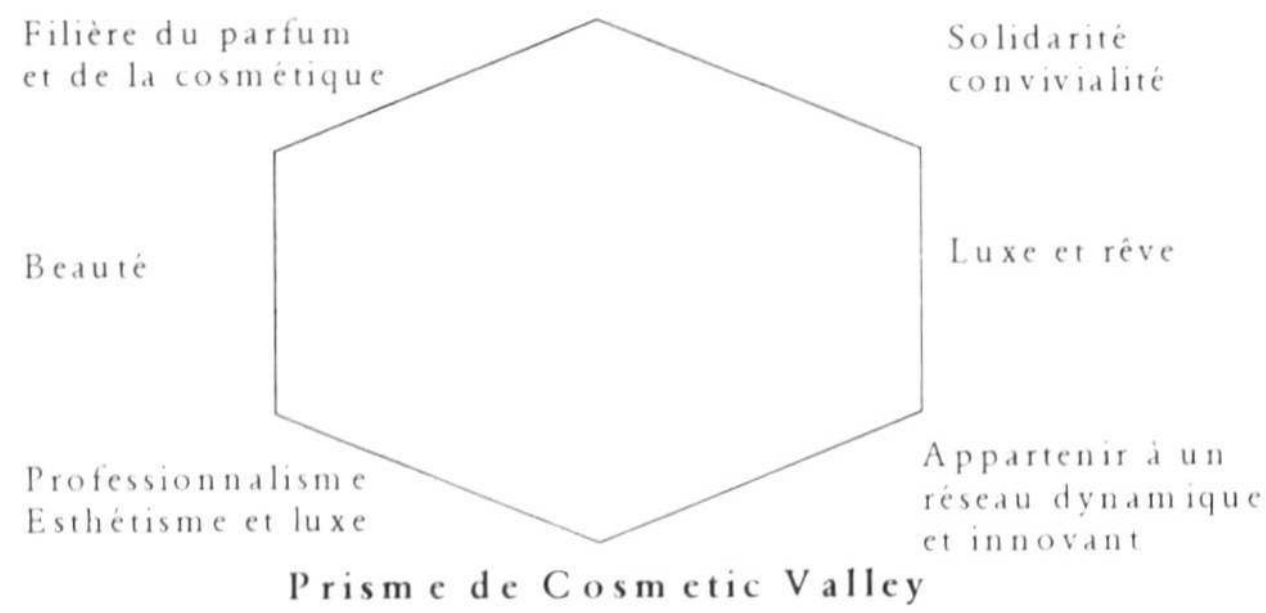




\section{Prisme de Cosmetic Valley}

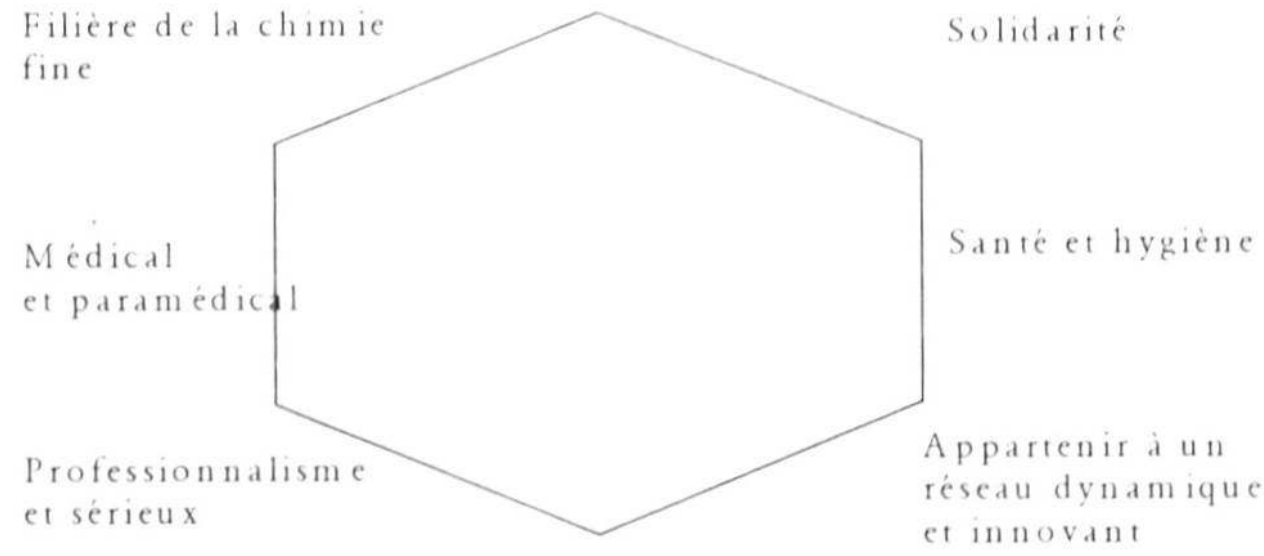

\section{Prisme de Polepharma}

\section{Prisme de Polepharma}

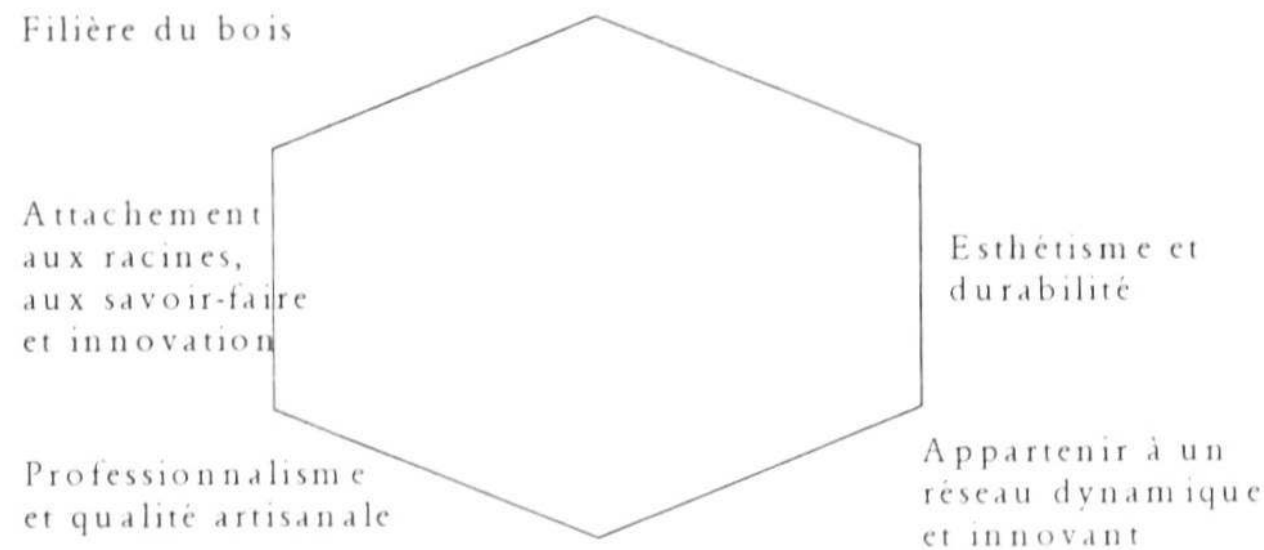

Prisme de Perchebois

\section{Prisme de Perchebois}

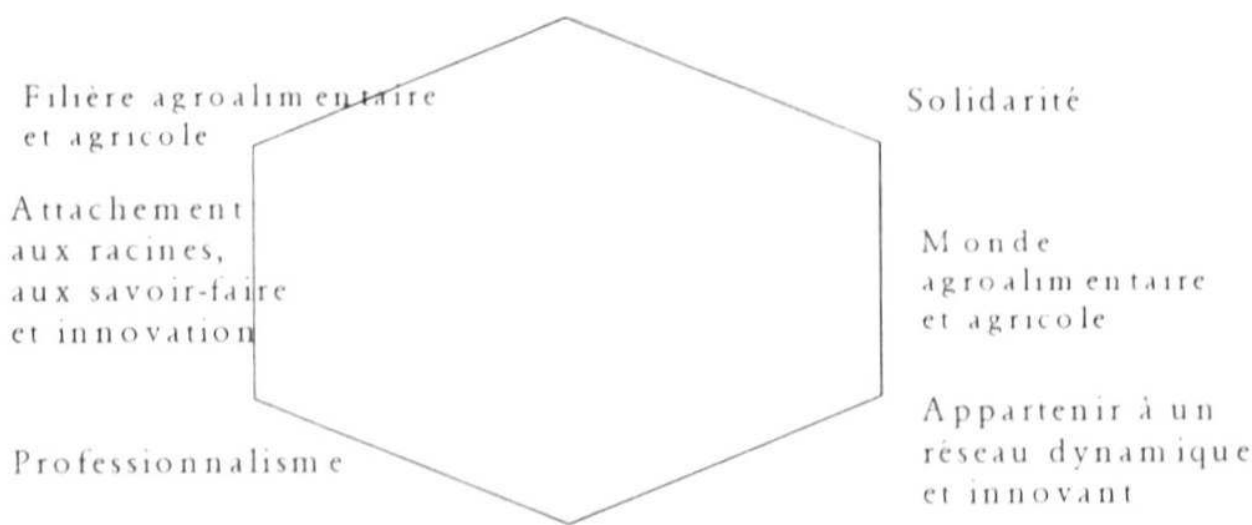

Prism e deAgrodynam ic 
Prisme de Agrodynamie être une rose. Mais le choix s'est focalisé sur la pivoine car "plus originale, plus esthétique, plus charnelle et en Asie, en Chine, elle symbolise le bonheur, l'odeur, le positif " nous explique Jean-Luc Ansel. Effectivement, les Péoniacées, que les chinois cultivent depuis le VIIe siècle, incarnent l'amour, la beauté féminine, la richesse, la prospérité et les honneurs. Cependant, un glissement de sens s'opère ici car une personne non avertie, non initiée a souvent tendance à voir en premier, une rose. De plus, le public connaît bien la rose de Grasse, célèbre dans le monde du parfum. Cette association reste, pour l'instant, inévitable. La couleur rose et/ou la représentation d'une fleur épanouie, la méconnaissance de la symbolique chinoise de la pivoine sont sans doute les raisons de cette confusion iconique. En effet, le passage de l'iconique au sème cultunsé reste, actuellement quelque peu délicat. Le code iconographique ne se réalise pas. Donc, la construction pragmatique de l'image du parfum, de la cosmétique, qui doit normalement fixer l'identité, l'image d'une marque ou dans notre cas, d'une association et que symbolise cette pivoine, ne s'opère pas totalement. couleur rassurante, rafraîchissante et humaine, symbolise la nature. Vert, la couleur de l'espérance, de la longévité et de la force. Le jaune fait référence aux céréales. En effet, cette couleur personnifie la terre fertile. Or, si nous pensons immédiatement aux céréales avec le jaune, les élevages de bétail apprécient eux aussi une terre fertile. Les grains de blé, signe logique et simple pour une grande région céréalière, comme la Beauce se retrouvent dans les supports de communication du pôle. La symbolique du blé nous ramène à l'union de Déméter avec Zeus. Un grain de blé avait été présenté comme hostie, contemplé en silence et il représentait Déméter, la déesse de la fécondité et l'initiatrice des mystères de la vie. La scène de l'époptie invoquait la pérennité des saisons, le retour 
des moissons et de sa résurrection en de multiples grains. Symbole d'immortalité, don de la vie, nourriture essentielle et primordiale. Ce code iconique se réalise facilement et n'entraîne aucune confusion ou incompréhension. Simple et efficace.

Le logo de Perchebois, une rosace et un panier fleuri, sculptés dans le bois sont les deux signatures qui caractérisent le mobilier percheron. On les retrouve sur tous les meubles ensembles ou séparés.

L'analyse qui suit, concerne uniquement le site de la Cosmetic Valley et son aspect esthétique, le reste de l'étude est en cours d'élaboration et fera l'objet d'une autre publication.

Dans la page d'accueil, l'image (la pivoine) se détache du flux visuel et joue sur ses propriétés graphiques et plastiques (texture, forme, couleur..). C'est ce que Souchier appelle « la mise en relation formelle par empreinte perceptive »

L'analyse de ce site montre une combinaison des visions classique et baroque. Nous utiliserons pour définir ces deux visions à la fois les travaux d'Heinrich Wölfflin et les cinq catégories qui permettent de reconnaître le classique du baroque et ceux de JeanMarie Floch sur l'empreinte et l'identité visuelles.

Linéaire vs pictural : la ligne tient une place importante dans la vision classique, « tout est dessiné ligne à ligne, de façon régulière et distincte; chaque ligne semble avoir conscience de sa beauté, mais aussi du fait qu'elle doit s'accorder avec ses compagnes ». Les lignes verticales et horizontales formées par le texte et l'insertion de la photographie illustrent la part classique du site. Dans la vision baroque, la primeur va aux masses et à l'enchaînement, à l'entrelacement (pétales de la pivoine et police d'écriture des titres).

Plans et profondeurs : chez les classiques, l'espace s'organise en plans distincts, parallèles entre eux, et frontaux par rapport à un observateur fixe. Tout s'organise en rectangles, parallèles entre eux. Dans le monde baroque, la profondeur entraîne une pluralité de points de vue et les figures ne sont pas côte à côte (le fond d'écran).

Forme fermée et forme ouverte : une forme est dite fermée lorsque l'image est «limitée en elle-même, réduite à une signification complète » Les droites et les angles du cadre du site luimême se retrouvent dans la barre de menu et celles des outils. En revanche, une forme ouverte se caractérisera par une image qui part dans tous les sens, en gardant cependant son unité. C'est le cas des formes souples, et sinuosidales du fond d'écran.

Multiplicité et unité : les divers éléments, distincts mais en accord entre eux, définiront la multiplicité du classique. Le principe d'unification qualifie le baroque, qui veut tout englober en une forme unique.

61 Clarté et obscurité : le traitement de la lumière diffère selon les visions classique et baroque. La lumière classique servira à une lisibilité immédiate des formes, tandis que la lumière baroque jouera sur la valeur et la désaturation chromatique, très nette entre la première page et le reste des pages du site.

62 À la fois baroque et classique, ce site manque de vie et ne reflète en aucun cas le dynamisme de l'association. Ce mélange de rigidité par les lignes et les rectangles rendent hiératique le site. Nous constatons, aussi un manque d'harmonie chromatique entre le logo et l'utilisation injustifiée de quatre couleurs pour l'écriture. Nous ne sommes plus, ni dans une vision classique, ni dans une vision baroque mais simplement dans une représentation hétéroclite qui valorise une totale absence de compétences et qui, malheureusement ne traduit absolument pas le dynamisme et la réussite de ce pôle. 
63 L'identité est un élément important d'une étude sur les groupements thématiques mais insuffisant pour commencer à élaborer de réelles conclusions sur ces quatre pôles de compétitivité euréliens, voire la possibilité de les comparer à d'autres filières du même thème. Je pense bien sûr à la filière du parfum de Grasse. Les pôles étudiés sont récents, la Cosmetic Valley va fêter ses dix ans d'existence, et pour certains d'entre eux, l'identité est un concept en complète élaboration. Cependant, tant qu'une filière vit, son identité évolue avec elle aussi.

À la suite de cette présentation, il sera intéressant de confronter identité et image de chaque adhérent, de chaque acteur économique pour voir les raisons qui poussent de grands groupes, de grands noms à adhérer à ce nouveau style de industriel et technologique. Quel(s) sens, quelle signification dans cet engagement?

\section{BIBLIOGRAPHIE}

FLOCH JM, Identités visuelles, collection Formes sémiotiques, PUF, 1995.

FLOCH J.-M., Sémioltique, marketing, communication, sous les signes les stratégies, Paris, PUF, 1990.

KAPFERER J .-N., Les marques, capital de l'entreprise. Créer et développer des marques fortes. Editions d'Organisation, 2002.

SOUCHIER E., « La publicité comme détournement du politique « , Communication et Langages $N^{\circ} 93$, Retz, 1992.

WOLFFLIN H., Principes fondamentaux de l'histoire de l'art, Brionne, Ed. Gérard Monfort, 1984.

\section{RÉSUMÉS}

Les pôles de compétitivité intéressent fortement la France, qui les a découvert tardivement, par rapport aux italiens. Les districts italiens existent depuis une trentaine d'années. Ceux de notre étude ont pour le plus âgé, dix ans d'existence et de fonctionnement. Une étude sur l'identité propre à chacun de ces pôles nous a semblé intéressante. Quelle identité et quels sont les signes qui permettent de la définir?

Poles of competitivences are of great interest to Prance which, compared to Italy, has discovered them rather behaledly. There have been Italian districts for about shirty years. The oldest case in our study has existed and been working for only ten years. The study of the characteristical identity of each pole has seemed a wathwhile under taking. What is their identity and what signs allons us to define it? 


\section{AUTEUR}

\section{FLORENCE HÉNON}

Maître de conférences à l'IUT de Chartres, Université d'Orléans et chargée de la communication de l'IUT. Sémioticienne de formation, elle s'intéresse à l'image dans les médias, surtout la télévision et dans les organisations. Mail : florence.henon@univorleans.fr 